\title{
Hallazgos tomográficos pulmonares y su asociación con niveles de linfocitos T CD4+ en pacientes portado- res del virus de inmunodeficiencia humana
}

\author{
Drs. Maryalejandra Mendoza de $\mathbf{S}^{(1)}$, Joanna Santeliz $C^{(1,2)}$, Fanny Ochoa $\mathrm{O}^{(1)}$, Ivy Contreras $V^{(1)}$, Fabiola Torres $\mathbf{S}^{(3)}$, \\ Mario Pérez $M^{(1)}$, Cleizer Altamiranda $V^{(1)}$. \\ 1. Instituto Autónomo Hospital Universitario de los Andes, Mérida, estado Mérida, Venezuela. \\ 2. Decanato de Ciencias de la Salud, Universidad Centroccidental "Lisandro Alvarado", Barquisimeto, estado Lara, Venezuela. \\ 3. Facultad de Medicina, Departamento de Imagenología, Universidad de los Andes, Mérida, estado Mérida, Venezuela.
}

CT pulmonary findings and their association with CD4 ${ }^{+} \mathrm{T}$ lymphocytes levels in human immunodeficiency virus carriers

\begin{abstract}
Respiratory diseases are frequent complications in HIV infection. High Resolution Computed Tomography (HRCT) has proven superior to conventional imaging techniques to establish a pulmonary disease diagnosis. A study was conducted in order to establish the association between tomographic pulmonary patterns and immune status of HIV-infected patients. We evaluated 35 patients with respiratory symptoms and /or abnormal chest radiograph. An association was observed between the presence of ground glass pattern and $P$ jirovecii infection. Likewise, a correlation between pulmonary histoplasmosis diagnosis and honeycomb pattern, lung cysts and nodules was established. Few correlation between tomographic patterns and CD4+ Tlymphocyte counts was observed. In summary, HRCT findings can predict certain types of infection; nevertheless, further studies are required to extrapolate this association to other noninfectious lung diseases in HIV-infected patients. Key words: CD4+, HIV, HRCT, Respiratory diseases.

Resumen: Las enfermedades respiratorias constituyen complicaciones frecuentes en la infección por VIH. La Tomografía Axial Computarizada de Alta Resolución (TACAR) ha demostrado ser superior a las técnicas de imagen convencionales para establecer diagnóstico de enfermedad pulmonar. Se realizó un estudio con la finalidad de establecer la asociación entre patrones tomográficos pulmonares y el estado inmunológico de pacientes $\mathrm{VIH}^{+}$. Se evaluaron 35 pacientes sintomáticos respiratorios y/o con radiografía torácica patológica. Se observó una asociación entre la presencia de patrón en vidrio esmerilado e infección por $P$ jirovecii. Asimismo, se observó una asociación entre diagnóstico de histoplasmosis pulmonar y patrón de panal de abejas, quistes pulmonares y nódulos. Se demostraron pocas correlaciones entre patrones tomográficos y conteo de linfocitos $T C D 4^{+}$. En conclusión, los hallazgos en la TACAR pueden predecir determinados tipos de infección requiriendo de más estudios para extrapolar esta asociación a otras enfermedades pulmonares no infecciosas en el paciente $\mathrm{VIH}^{+}$.

Palabras clave: Enfermedades respiratorias, CD4+, TACAR, VIH.
\end{abstract}

Mendoza M. Hallazgos tomográficos pulmonares y su asociación con niveles de linfocitos $T C D 4^{+}$en pacientes portadores del virus de inmunodeficiencia humana. Rev Chil Radiol 2011; 17(2): 61-66.

Correspondencia: Dra. Maryalejandra Mendoza de Sifontes / aleynico@hotmail.com

Trabajo recibido el 04 de febrero de 2011, aceptado para publicación el 31 de marzo de 2011.

\section{Introducción}

Tanto la infección por el virus de inmunodeficiencia humana $(\mathrm{VIH})$ como su expresión clínica, el síndrome de inmunodeficiencia adquirida (SIDA) representa un importante problema de salud pública, ya que ambas entidades se expresan en proporciones epidémicas.
Desde la aparición del VIH cerca de 60 millones de personas se han infectado con él y 25 millones de personas han fallecido por causas relacionadas con la infección. En América Latina el total de nuevas infecciones para el año 2008 se cifró en 170.000 y hoy en día se considera que en la región 2 millones 
de personas viven infectadas con el virus. Según las mismas estimaciones, 77.000 personas han fallecido por causas relacionadas con el $\mathrm{VIH}$ aun cuando aparentemente en Latinoamérica la epidemia se ha estabilizado ${ }^{(1)}$.

En lo que respecta a la evolución natural de la enfermedad, se considera excepcional que una persona infectada por VIH no presente en el transcurso de la enfermedad alguna afectación importante del aparato respiratorio; más del $70 \%$ de los pacientes presentan alguna complicación pulmonar tanto de índole infecciosa como no infecciosa ${ }^{(2,3)}$. En cerca del $90 \%$ de las necropsias realizadas a individuos seropositivos, hay participación pulmonar en el proceso que lleva a su fallecimiento ${ }^{(4)}$.

La enfermedad pulmonar condicionada por la infección por VIH tiende a presentar multiplicidad de facetas, atipicidad de cuadros clínicos e imágenes radiológicas inespecíficas, lo cual supone una mayor dificultad para el diagnóstico etiológico de la patología respiratoria en estos pacientes. En un considerable porcentaje de los individuos con VIH/SIDA se puede lograr un diagnóstico etiológico con la combinación de hallazgos clínicos, imagenológicos e inmunológicos. Sin embargo, en un $5-10 \%$ de los pacientes seropositivos los hallazgos paraclínicos, radiológicos y aún patológicos pueden ser inespecíficos, lo que dificulta aún más el diagnóstico definitivo de estas enfermedades ${ }^{(5)}$.

Varios estudios han evidenciado que la tomografía axial computarizada (TAC), particularmente la tomografía de alta resolución (TACAR), puede mostrar anormalidades parenquimatosas pulmonares en pacientes con radiografías normales y es superior a esta técnica convencional a la hora de diagnosticar o excluir enfermedad pulmonar ${ }^{(6-8)}$. Los hallazgos de la TAC y TACAR de Tórax facilitan el monitoreo de la enfermedad, permiten establecer diagnósticos diferenciales, así como determinar la presencia de posibles complicaciones; además de ello, la TAC es un instrumento especialmente útil para guiar procedimientos que permitan la toma de muestras en los pacientes seropositivos ${ }^{(5-7)}$. Por lo tanto, la TAC y la TACAR actualmente representan un elemento a incorporar en el arsenal diagnóstico del paciente portador del VIH con sospecha de patología respiratoria.

El conteo de la población de linfocitos $\mathrm{TCD}^{+}{ }^{+}$es el reflejo del estado inmune del paciente seropositivo. Estudios multicéntricos han establecido una clara asociación entre el descenso de linfocitos T CD4 ${ }^{+} y$ el mayor riesgo de enfermedades oportunistas tanto sistémicas como respiratorias ${ }^{(9)}$. En vista de lo anteriormente mencionado, el objetivo de este estudio es evaluar la asociación entre los hallazgos en la TACAR torácica y el estado inmunológico del paciente $\mathrm{VIH}^{+}$ basados en los niveles de linfocitos T CD4+.

\section{Materiales y métodos}

Se incluyeron pacientes mayores de 18 años, de ambos sexos, sintomáticos respiratorios, identificados como seropositivos para VIH con o sin manifestaciones de SIDA que acudieron al Instituto Autónomo Hospital Universitario de los Andes (Mérida, estado Mérida, Venezuela). Se utilizó la guía de recomendaciones a médicos en investigación médica que involucra seres humanos según la Declaración de Helsinki modificada en 2005. El estudio fue aprobado por el Comité Ético de dicho hospital y se obtuvo el consentimiento informado de todos los pacientes que participaron en la investigación. Se elaboró una historia clínica detallada, radiografía postero-anterior de tórax y TACAR de tórax. Las imágenes obtenidas fueron evaluadas independientemente por dos médicos radiólogos y se catalogaron los hallazgos tomográficos de acuerdo a la nomenclatura establecida internacionalmente por el Nomenclature Committee of the Fleishner Society ${ }^{(10-12)}$.

Se recolectó una muestra de sangre venosa para el contaje de linfocitos $\mathrm{T} \mathrm{CD}^{+}$y $\mathrm{CD} 8^{+}$previo a la realización de estudios invasivos. A fin de determinar la etiología de la patología pulmonar se realizó fibrobroncoscopía para la obtención de lavado broncoalveolar (LBA), cepillado bronquial protegido y biopsia transbronquial siguiendo las recomendaciones de la Sociedad Americana del Tórax (ATS) y de la Sociedad Respiratoria Europea ${ }^{(13,14)}$. La biopsia transbronquial se realizó posterior a la recolección del LBA, tomándose 4 a 5 muestras de tejido de la zona afectada, las cuales fueron colocadas en una solución de formalina al $10 \%$ para su ulterior procesamiento histológico. Las muestras fueron coloreadas con Hematoxilina-Eosina, ácido Periódico de Schiff (PAS), Tricrómico y Ziehl Neelsen. Se utilizó la coloración de metenamina de Plata (Grocott) para la identificación histopatológica de hongos.

Se realizó cultivo bacteriológico y micológico tomando una muestra de $15 \mathrm{cc}$ de lavado broncoalveolar para cada cultivo. El cultivo bacteriológico se consideró positivo si se detectó crecimiento de más de $10.000 \mathrm{UFC} / \mathrm{ml}$ de LBA. Asimismo, se realizaron pruebas serológicas tipo IgG e lgM para la determinación de infección por bacterias atípicas (Mycoplasma pneumoniae y Chlamydia pneumoniae), hongos $(H$. Capsulatum, Aspergillus sp, $P$. Brasiliensis, Candida $s p, C$. neoformans y $C$. inmitis) e infección por toxoplasma y/o citomegalovirus.

El análisis estadístico se realizó utilizando el programa SPSS versión 15.0 para Windows. Las variables cualitativas se caracterizaron mediante frecuencias simples y porcentajes. Para establecer asociación entre los rangos de linfocitos $\mathrm{T} \mathrm{CD4}^{+}$, síntomas respiratorios y diagnóstico de enfermedad pulmonar con patrones tomográficos se utilizaron cuadros de doble entrada aplicándose el análisis estadístico con $X^{2}$. Para establecer la asociación entre valores de 
linfocitos T CD4+ y los patrones tomográficos con la aparición de determinada enfermedad pulmonar se aplicó el Test de Fischer para probar independencia entre las variables; una vez probada su dependencia se aplicó Test del Coeficiente de phi.

\section{Resultados}

Treinta y cinco pacientes fueron incluidos en el estudio. La edad promedio de los participantes fue de $39 \pm 2$ años (rango: 20-77 años). Veintinueve pacientes $(83,9 \%)$ pertenecían al género masculino. En relación al uso de terapia antirretroviral de gran actividad (HAART) al momento de ingresar al estudio $51,4 \%$ de los pacientes no la recibían; así mismo, ningún paciente recibía profilaxis para $P$. jirovecii o M. tuberculosis.

Al evaluar la presencia o no de infección pulmonar, 30 de los 35 pacientes (85.7\%) presentaron cultivos microbiológicos o muestras histopatológicas positivas para bacterias y/o hongos; estas infecciones fueron documentadas de la siguiente manera: 8 infecciones exclusivamente micóticas, 5 de ellas por $P$. jirovecii, 2 por $H$. capsulatum y Candida $s p$ (invasiva) en 1 paciente; 4 infecciones de etiología estrictamente bacteriana por $S$. viridans, $M$. catarrhalis, $P$. aeruginosa, $H$. influenzae (una para cada caso) y 19 pacientes $(63,3 \%)$ presentaron infecciones mixtas (micóticas y bacterianas). En aquellos pacientes con infecciones polimicrobianas, las bacterias aisladas en orden de frecuencia fueron $S$. pneumoniae, $S$. viridans, M. catarralis, $P$. aeruginosa, E. coli, $S$. aureus, C. pneumoniae, K. pneumoniae, $H$. influenzae, S. maltophila y C. luteola.

La enfermedad pulmonar de origen viral fue diagnosticada en 4 casos (11.42\%), siempre asociada a un agente bacteriano, y se encontró definida en todos los casos por la presencia de citomegalovirus. La etiología no infecciosa se evidenció en tres (8,57\%) pacientes, en dos de ellos dada por neumonía intersticial no especifica (NINE) y en el otro caso se documentó carcinoma pulmonar.

En relación a las subpoblaciones de linfocitos en sangre periférica se observó que el promedio de linfocitos T CD4 $4^{+}$y CD8 ${ }^{+}$en sangre periférica fue 315 \pm 49 células $/ \mu$ y $869 \pm 94$ células $/ \mu \mathrm{l}$, respectivamente. En promedio, los linfocitos $\mathrm{T} \mathrm{CD} 4^{+}$representaron el $23 \% \pm 3 \%$ del total de linfocitos en sangre periférica mientras que los linfocitos $\mathrm{T} \mathrm{CD8} 8^{+}$representaron el $62 \% \pm 4 \%$. El promedio de la relación $\mathrm{CD} 4^{+} / \mathrm{CD} 8^{+}$fue de $0,4 \pm 0,08$ y la media de la carga viral plasmática para el grupo estudiado fue de $78.830 \pm 24.795$ copias $/ \mathrm{ml}$. Según la categorización establecida por el Centers for Disease Control (CDC) en relación al contaje de linfocitos T CD4+ se observó que el 31\% de los pacientes presentó niveles mayores a 500 células/ $\mu \mathrm{l}$, (Grupo 1), 25\% tenía niveles entre 200-499 células $/ \mu \mathrm{l}$ (Grupo 2) y $44 \%$ de los casos presentaron recuentos menores a 200 células $/ \mu \mid$ ".

Trece por ciento de los pacientes presentaron radiografía torácica normal o casi normal (presencia de granulomas calcificados residuales); cuando las radiografías postero-anterior de tórax se mostraron patológicas predominó la presencia de patrón intersticial principalmente reticular. El derrame pleural estuvo presente en dos pacientes asociado a infiltrado alveolar en un caso y a infiltrado intersticial reticular en el otro.

En las treinta y cinco TACAR examinadas se identificaron 110 lesiones del parénquima pulmonar, evidenciándose una importante superposición de patrones. Se analizó la frecuencia individual de cada uno de los hallazgos patológicos en la TACAR, hallándose en primer lugar el engrosamiento septal $(18,2 \%)$, seguido de engrosamiento peribroncovascular (16,4\%), vidrio esmerilado y bandas subpleurales ( $13,6 \%$ cada una), nódulos y consolidación (10,9\% cada una), presencia de quistes o bullas $(6,4 \%)$, aumento de la reticularidad y bronquiectasias ( $4,5 \%$ cada una) y finalmente la presencia de panal de abejas (0,9\%) (Figura 1). Al estudiar la ubicación de los infiltrados tomográficos torácicos se observó que muy frecuentemente la afectación correspondió a más de un segmento pulmonar; se aprecia predominio de la distribución universal en $36,3 \%$ de los casos, seguido por la ocupación bibasal en $30 \%$, luego la afectación en ambos vértices en $5,5 \%$ y en todo el pulmón izquierdo 2,7\%. Cuando se observó la lesión limitada a un lóbulo pulmonar, el lóbulo inferior izquierdo se afectó en 12,7\% mientras que los lóbulos superior derecho e izquierdo e inferior derecho se encuentran comprometidos en una pequeña proporción.

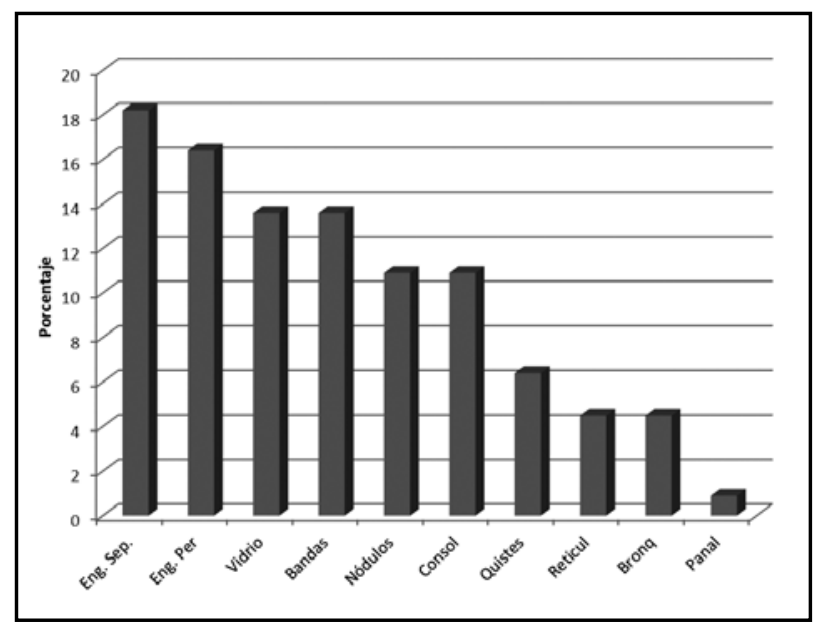

Figura 1. Porcentaje y tipos de Infiltrados en la TACAR en pacientes portadores de VIH/SIDA incluidos en el estudio. *Eng. Sep: Engrosamiento septal; Eng. Per: Engrosamiento peribroncovascular; Vidrio: Vidrio esmerilado; Consol: Consolidación; Reticul: Aumento de la reticularidad; Bronq: Bronquiectasias. 
Se identificaron 62 alteraciones tomográficas no parenquimatosas. En $38,7 \%$ de los pacientes se observaron adenopatías $>10 \mathrm{~mm}$ y de localización mayoritariamente mediastínicas; le siguen en frecuencia las calcificaciones en $32,3 \%$ de los casos, en mayor proporción hiliares y pocas parenquimatosas; el engrosamiento pleural fue observado en $22,6 \%$ de los pacientes (bilateral en su mayoría), seguido por derrame pleural en $4,8 \%$ (bilateral y leve) y por último neumotórax marginal en 1,6\% (Figura 2). En todos los casos en las que estas alteraciones fueron descritas, las mismas se asociaron al menos a un hallazgo patológico parenquimatoso.

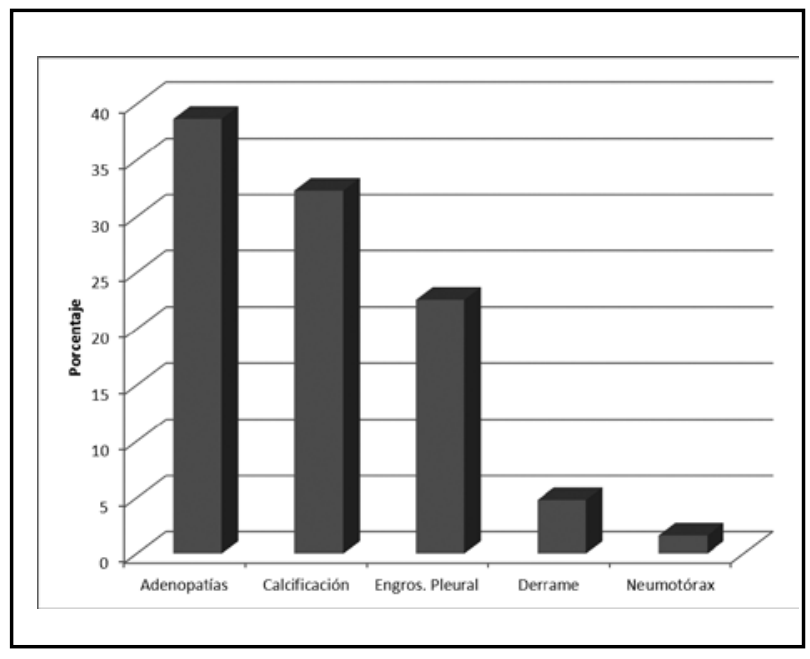

Figura 2. Porcentaje de hallazgos tomográficos no parenquimatosos en pacientes portadores de VIH/SIDA incluidos en el estudio.

Al asociar los hallazgos tomográficos con los agentes etiológicos aislados se evidenció una asociación estadísticamente significativa entre la presencia de patrón en vidrio esmerilado (Figura 3) y la enfermedad pulmonar de etiología bacteriana en general $(p=0,008)$ y esta asociación también fue válida para la infección micótica específicamente con la infección por $P$. jirovecii $(\mathrm{p}=0,02)$. Asimismo, se observó una correlación significativa entre la presencia de histoplasmosis pulmonar y alteraciones tomográficas tales como panal de abejas $(p=0,002)$, quistes pulmonares $(p=0,04)$ y nódulos $(p=0,01)$. El resto de los diagnósticos de enfermedad pulmonar no parecen asociarse a ningún infiltrado tomográfico en particular.

En relación a la asociación entre patrones tomográficos y niveles de linfocitos T CD4+ se observó una correlación con significancia estadística entre el patrón de vidrio esmerilado y valores de CD4 ${ }^{+}$ superiores a 500 células $/ \mu \mathrm{l}\left(\mathrm{X}^{2}=4,21, \mathrm{p}=0,04\right)$ y en rango de 50 a 200 células $/ \mu \mathrm{l}\left(X^{2}=5,18, p=0,02\right)$, sin embargo, esta asociación se mantiene a cualquier conteo celular.
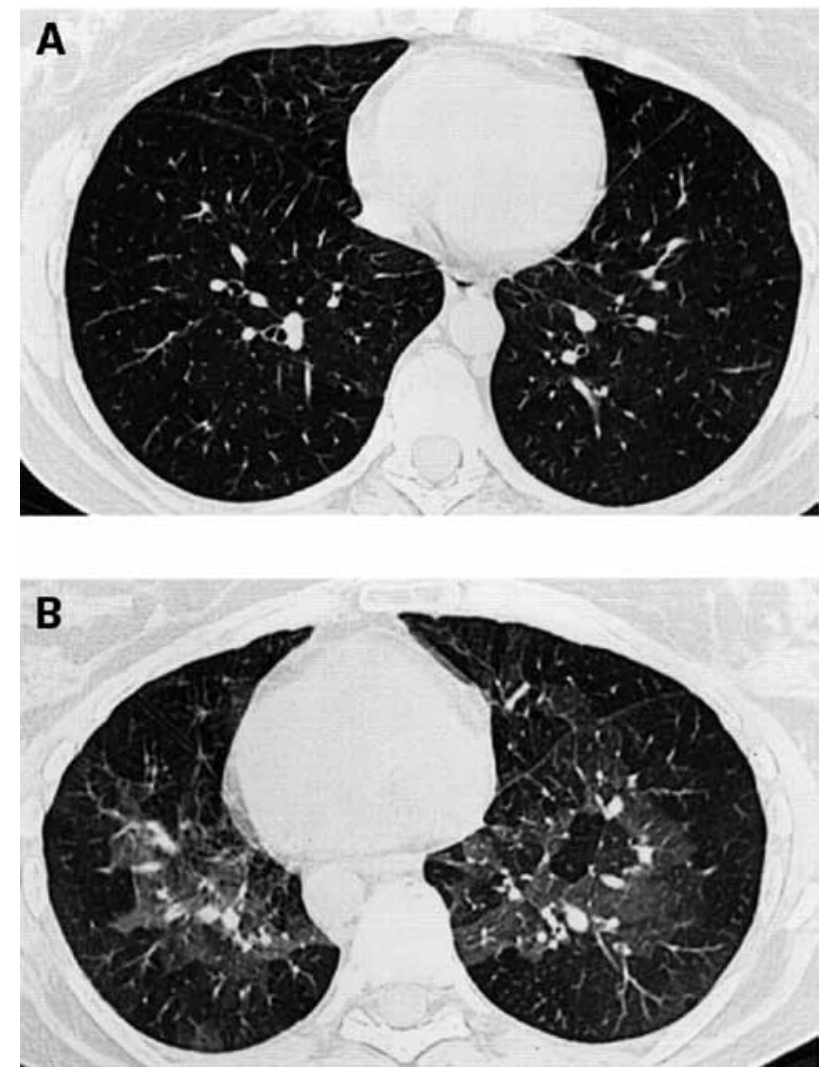

Figura 3. TACAR de tórax normal (A) y de paciente donde se observa opacidad en vidrio esmerilado de distribución difusa (B).

\section{Discusión}

La radiografía de tórax convencional es la modalidad de imagen empleada convencionalmente para la evaluación inicial de los pacientes VIH positivos con síntomas respiratorios. En la muestra estudiada, 13\% de los pacientes presentaron radiografías de tórax normales o casi normales y aunque estudios internacionales han evidenciado que esta población tiende a presentar enfermedad respiratoria con imágenes radiológicas normales o no específicas ${ }^{(15)}$, no se ha reportado a qué porcentaje de pacientes corresponde este fenómeno.

Al evaluar las imágenes patológicas en las radiografías torácicas se evidenció un amplio predominio del patrón intersticial de tipo reticular, solo o acompañado por micronódulos y/o patrón alveolar; estos hallazgos son frecuentemente comentados por diversos autores en el diagnóstico de la enfermedad infecciosa pulmonar en el paciente seropositivo, sin embargo, resultan inespecíficos para la identificación etiológica de la patología en particular ${ }^{(16)}$.

La tomografía de tórax de alta resolución mostró una mayor cantidad de alteraciones del parénquima pulmonar, aun en pacientes con radiografías normales o casi normales, situación que ha sido citada en 
varias publicaciones $^{(6,7)}$. Los hallazgos más frecuentes correspondieron a densidades reticulares lineales (evidenciándose en más del $50 \%$ de los casos); las imágenes tomográficas producto del aumento de la densidad pulmonar continúan en el orden de frecuencia dadas mayormente por la presencia de vidrio esmerilado y luego por la consolidación tanto local como difusa. Las densidades nodulares difusas (micronódulos en todos los casos) fueron advertidas en tercer lugar de frecuencia; culminando con las imágenes condicionadas por la disminución de la densidad del parénquima pulmonar (quistes, bullas y/o bronquiectasias).

Muy pocos autores hacen descripciones simples de los hallazgos tomográficos más usuales en la población VIH positiva. Guillemi y colaboradores evaluaron las lesiones inesperadas en TACAR de pacientes en fase SIDA con radiografía torácica no patológica observando en $60 \%$ de los sujetos alteraciones parenquimatosas muy similares a las observadas en nuestra población, pero en distinto orden de frecuencia ${ }^{(17)}$. Las alteraciones por él descritas son en mayor proporción lesiones quísticas y enfisematosas, seguidas por nódulos, vidrio esmerilado, consolidación y opacidades lineales, no haciendo referencia a las etiologías que las condicionaron.

Las lesiones parenquimatosas se distribuyeron de forma extensa en la TACAR (se localizaron en más de un lóbulo pulmonar) en una proporción mayor al $75 \%$, presentándose mayormente con distribución universal, seguidas por un componente bibasal. De forma curiosa, cuando las lesiones se observaron limitadas a un solo segmento pulmonar, el lóbulo inferior izquierdo fue por mucho la región más frecuentemente afectada, no conociéndose explicación alguna en la literatura para este hecho.

Más allá de las lesiones parenquimatosas, la TACAR resultó ser muy útil para demostrar alteraciones mediastinales y pleurales que pasaron inadvertidas en casi la totalidad de las radiografías simples. Con los hallazgos aquí descritos se ratifica lo descrito por Mason y colaboradores y comentado por diversos autores después de él: "la tomografía es un instrumento muy útil para el estudio de patología mediastínica, pleural, diafragmática y de pared torácica en la población en general, pero aún de mayor utilidad en la población VIH positiva, donde la clínica y la radiología tienden a ser inespecíficas"(5).

De forma incidental se logró observar una falta de asociación entre hallazgos tomográficos y sintomatología respiratoria aun demostrándose enfermedad pulmonar, hecho que ha sido mencionado por otros autores, lo cual debe alertar al médico en la búsqueda de patología pulmonar aun en pacientes que refieran ser asintomáticos.

La asociación existente entre alteraciones tomográficas y enfermedades pulmonares en la población VIH positiva ha sido bien establecida por distintos autores, quienes en la mayoría de los casos identifican más de un tipo de infiltrado como predictor de un determinado diagnóstico etiológico. Pese a que en nuestra investigación se observaron 7 patologías distintas relacionadas a la infección por el $\mathrm{VIH}$ de causa infecciosa y no infecciosa, no fue posible establecer una relación con las anormalidades en TACAR para la mayoría de ellas; es así, que sólo pudo correlacionarse la presencia de infección por $P$. jirovecii con el patrón en vidrio esmerilado (asociación ya comentada por varios investigadores), y en segundo lugar los nódulos, quistes y panal de abeja con histoplasmosis (relación también descrita por otros autores) ${ }^{(2,15,18-21)}$.

Realizando una amplia revisión bibliográfica fueron escasos los trabajos hallados donde se establecen asociación entre los hallazgos tomográficos y conteo celular linfocitario en pacientes $\mathrm{VIH}^{+}$. Guillemi y colaboradores asoció la presencia de lesiones pulmonares en TACAR con el valor de la media de linfocitos T $\mathrm{CD}^{+}$no encontrando diferencia estadística con los controles (pacientes VIH sin alteración tomográfica) ${ }^{(16)}$. Asimismo, Jasmer y colaboradores asociaron sólo la presencia de nódulos en la TAC de Tórax con niveles de linfocitos T CD4+ para la predicción de enfermedad respiratoria, no siendo estadísticamente significativa esta asociación para infección bacteriana u oportunista, sólo para tuberculosis pulmonar y conteo de linfocitos $\mathrm{T}$ CD4+ inferior a 50 células $/ \mathrm{mm}^{(3)}$.

En la presente investigación se asociaron los hallazgos patológicos de la TACAR torácica con los niveles de linfocitos $\mathrm{TCD} 4^{+}$en base a la categorización clínica establecida por el CDC encontrando una pobre correlación entre ambas variables; sólo fue positiva para el infiltrado en vidrio esmerilado. Asimismo se pudo demostrar una fuerte dependencia de la infección por $P$. jirovecii con la presencia de este mismo patrón independiente de los valores de linfocitos $T$ $\mathrm{CD}^{+}$. Aunque la neumocistosis es reconocida como una enfermedad oportunista, fue evidente su hallazgo en pacientes con conteo a 200 células $/ \mu \mathrm{l}$; aunque no es posible explicar este hallazgo a la luz de los estudios reportados en la literatura, se puede atribuir a la ausencia de quimioprofilaxis para este agente en específico en muestra evaluada.

De la misma asociación se pudo constatar que la opacidad en vidrio esmerilado y el conteo celular de linfocitos inferior a 50 células $/ \mu \mathrm{l}$ se correlacionan con fuerza con la presencia de infección pulmonar, bacteriana en general; si bien este patrón tomográfico corresponde a un aumento de la densidad pulmonar la falta de consolidación observada pudiese ser una expresión de la deficiencia superior en estos sujetos, que imposibilita el desarrollo una respuesta inflamatoria completa.

En definitiva, podemos concluir que existe una asociación entre la presencia de patrón en vidrio esmerilado y enfermedad pulmonar de etiología mi- 
cótica específicamente con la infección por $P$. jirovecii a cualquier conteo de linfocitos $\mathrm{T} \mathrm{CD}^{+}$así como la infección de causa bacteriana en pacientes profundamente inmunodeprimidos. Asimismo se observó una asociación entre la presencia de histoplasmosis pulmonar y el patrón de panal de abejas, quistes pulmonares y nódulos. A pesar de que no se demostró una correlación entre los patrones tomográficos y el conteo de linfocitos T CD4+ para el resto de las patologías respiratorias encontradas en la población estudiada, nuestros hallazgos sugieren que TACAR puede predecir la presencia de determinados tipos de infección en pacientes seropositivos, requiriéndose estudios adicionales para extrapolar esta asociación a otras enfermedades pulmonares vinculadas a la infección por el VIH. Es de acotar que en base a los resultados presentados en este estudio no es posible realizar inferencias acerca del estado inmunológico del paciente $\mathrm{VIH}^{+}$en base a la ausencia o presencia de hallazgos patológicos en la TACAR de tórax, hecho que fue reflejado ampliamente en la muestra estudiada.

\section{Agradecimientos}

Expresamos nuestra gratitud al Dr. Luis Godoy por su colaboración en la interpretación imaginológica, a la Dra. Carmen Fuenmayor por el estudio histopatológico, a la Dra. Celina Pérez de Salazar por el análisis micológico de las muestras y al Licenciado Henry Andrade por su apoyo en el análisis estadístico, al Dr. José Cova y a la Lic. Lérida Borges por los estudios inmunológicos. Asimismo, agradecemos el apoyo del personal de Enfermería del Servicio de Neumonología y Cirugía de Tórax y del personal del Laboratorio de Hormonas del IAHULA. Proyecto M-972-09-07-C financiado por el Centro de Desarrollo Científico, Humanístico y Tecnológico (CDCHT) de la Universidad de los Andes, Mérida, estado Mérida, Venezuela.

\section{Bibliografía}

1. ONUSIDA. Informe sobre la epidemia mundial de SIDA 2008. [En línea] Ginebra. Diciembre 2006 [Citado el 02 de marzo de 2008]. Disponible en: URL:http:// www.unaids.org/en/Knowledge/HIVData/EpiUpdate/ EpiUpdArchive/2009/default.asp.

2. Gassiot C, Pino P, Ramos M. Neumopatías asociadas al Sida. Acta Médica 2000; 9(1-2): 73-89.

3. Benito N, Moreno-Camacho A, Torres A. Pulmonary infiltrates in HIV patients in the highly active antiretroviral therapy era. Clinical Pulmonary Medicine Nov 2008; 15(6): 313-324.

4. Miller R. HIV-associated respiratory disease. Lancet 1996; 348: 307-312.

5. Mason A, Muller N. The role of computed tomography in the diagnosis and management of human immunodeficiency Virus (HIV)-related pulmonary disease. Seminars in ultrasound CT an MRI. April 1998; 19(2): 154-66.

6. Kang E, Staples C, McGuinness G, Primack S, Muller $\mathrm{N}$. Detection and differential diagnosis of pulmonary infections and tumors in patient with AIDS: value of chest radiography versus CT. American Journal of Roentgenology 1996; 166: 15-20.

7. Hartman T, Primack S, Muller N, Staples C. Diagnosis of thoracic complications in AIDS: accuracy of CT. American Journal of Roentgenology 1994; 162: 547554.

8. Watkins B, Klotman, Gallo R. Human immunodeficiency viruses. En: Principles and practice of infectious diseases. Ed: GL Mandell, JE Douglas, R Dolin. Churchill Livingstone. 6 ed. New York: 1995. p. 1590-1606.

9. Hanson D, Chu K, Farizo M, Ward J. Distribution of $\mathrm{CD}^{+} \mathrm{T}$ lymphocytes at diagnosis of acquired immunodeficiency syndrome-defining and other human immunodeficiency virus-related illnesses. Archives of Internal Medicine 1995; 155: 1537-1546.

10. Pedrosa J. Aspectos generales de la TACAR. En: Pedrosa J, Tomografía de alta resolución en Tórax. $2^{\circ}$ edición. México 2004. p 17-30.

11. Naidich D. Aspectos técnicos de la tomografía computarizada de alta resolución. En: Webb R, Muller N, Naidich D. Alta Resolución en TC de Pulmón. $3^{a}$ ed. España. Marban Libros. 2003. p 1-47.

12. Gotway M. High-resolution CT of the lung: patterns of disease and differential diagnoses. Radiologic Clinics of North America 2005; 43(5): 513-542.

13. American Thoracic Society. Clinical role of bronchoalveolar lavage in adults with pulmonary disease. Am Rev Respir Dis 1990; 142: 481-486.

14. European Society of Pneumology Task Group on BAL. Technical recommendations and guidelines for bronchoalveolar lavage (BAL). Eur Respir J 1989; 2: 561-585.

15. Gruden J, Huang L, Turner J, Webb J, Merrifield C, Stansell $\mathrm{J}$ et al. High-Resolution CT in the evaluation of clinically suspected Pneumocystis carinii pneumonia in AIDS patients with normal, equivocal or nonspecific radiographic findings. American Journal of Roentgenology 1997; 169: 967-975.

16. King L, Padley P. Imaging of the thorax in AIDS. Imaging 2002; 14: 60-76.

17. Guillemi S, Staples C, Hogg J, Lawson L, Schechter M, Montaner J. Unexpected lung lesion in high resolution computed tomography (HRTC) among patients with advanced HIV disease. European Respiratory Journal 1996; 9: 33-36.

18. Gold J, Rom W, Hakbin T. Significance of Abnormal Chest Radiograph Findings in patients with HIV-1 Infection without respiratory symptoms. CHEST 2002; $121 ; 1472-1477$.

19. Richards P, Riddell L, Reznek R, Armstrong P, Pinching A, Parkin J. High resolution computed tomography in HIV patients with suspected Pneumocystis carinii pneumonia and a normal chest radiograph. Clinical Radiology 1996; 51:689-693.

20. White $S$. Acutte lung infections in normal and inmunocompromised host. Radiologic Clinics of North America 2006; 44 (2): 295-316.

21. Nyamande K, Lalloo U, Vawda F. Comparison of plain chest radiography and high-resolution $C T$ in human inmunodeficiency virus infected patients with communityacquired pneumonia: a sub-Saharan Africa study. The British Journal of Radiology 2007; 80: 302-306. 